\title{
Lattice dynamics of single-walled boron nitride nanotubes
}

\author{
Valentin N. Popov* \\ Physics Department, University of Antwerp (RUCA), 2020 Antwerp, Belgium \\ (Received 27 September 2002; revised manuscript received 9 December 2002; published 12 February 2003)

\begin{abstract}
The phonon dispersion of single-walled boron nitride nanotubes is calculated within a valence shell model of the lattice dynamics. The model parameters are derived from fittings to the measured phonon dispersion of hexagonal boron nitride and available optical data. The calculated phonon dispersion curves for the tubes $(10,10),(17,0)$, and $(15,4)$ are presented. An analysis of the vibrational patterns of the zone-center phonons is given. The results for the phonon dispersion, zone-center phonons, and nonresonant Raman intensity can be used for the needs of characterization of boron nitride nanotube samples by Raman scattering and infrared spectroscopies.
\end{abstract}

DOI: 10.1103/PhysRevB.67.085408

PACS number(s): 63.22. $+\mathrm{m}, 78.30 .-\mathrm{j}$

\section{INTRODUCTION}

Boron nitride nanotubes (BNNTs) were theoretically predicted to be stable structures with an electronic band gap, independent of their radius and chirality. ${ }^{1}$ Extensive firstprinciples calculations showed that they are wide-gap semiconductors with an energy gap of $\sim 5.5 \mathrm{eV}$, and that their stability is related to the buckling effect. ${ }^{2}$ The possibilities for technological applications of BNNTs motivated the development of various methods for their production: arc discharge, ${ }^{3-6}$ laser ablation, ${ }^{7,8}$ and chemical vapor deposition. ${ }^{9}$ The structural study of the samples performed by high-resolution transmission electron microscopy ${ }^{10-12}$ revealed that synthesized BNNTs had a hexagonal (h-) BN stacking of zigzag type layers, ${ }^{10} A B A B$ hexagonal stacking of zigzag and armchair layers, ${ }^{11}$ rhombohedral (r-) BN stacking $(A B C A B C \ldots)$ and a near zigzag arrangement. ${ }^{12} \mathrm{By}$ an $\mathrm{X}$-ray diffraction analysis of catalytically grown BNNTs, a stacking of h-BN layers with two-layer repeat units and r-BN layers with three-layer repeat units was measured. ${ }^{13} \mathrm{Re}-$ cently, it was possible to produce by laser-ablation BNNTs samples which were composed mostly of single-walled BNNTs of predominantly zigzag type with diameter of 1.6 $\mathrm{nm}$ and well-crystallized bundles of about ten tubes with tube diameter of $1.4 \mathrm{~nm}^{.14}$

The characterization of the synthesized BNNTs can be accomplished by means of Raman scattering and infrared spectroscopies as faster, cheaper, and nondestructive methods. The observed features in the Raman and infrared spectra of BNNT samples can be assigned to definite tube vibrational modes by using theoretical predictions for these modes within a lattice-dynamical model. As far as it is known to the author, no calculations of the phonons of BNNTs have so far been reported, the theoretical efforts being directed mainly to electronic band structure calculations , $^{1,2}$ and study of the mechanical properties of the BNNTs. ${ }^{15-18}$ The dynamical problem for BNNTs can be solved by the zone-folding method or by direct simulations for a particular tube. Since the former approach has certain drawbacks, ${ }^{19}$ it is preferrable to use the latter one.

Here a valence shell model for the lattice dynamics of single-walled BNNTs (hereafter, simply BNNTs) with parameters obtained from fitting to experimental phonon dis- persion and measured optical modes frequencies of h-BN is proposed (Sec. II). This model is symmetry adapted, and is based on a unit cell of two atoms only. In Sec. III, the results of the calculations of the phonon dispersion for three BNNTs are presented. Analysis of the zone-center phonons is given. The derived radius dependence of the frequency of some of the optical phonons for all tubes with radii between 5 and 25 $\AA$ is also given. Finally, the nonresonant Raman spectra for the considered three tubes is calculated within a bondpolarizability model. The paper ends with conclusions (Sec. IV).

\section{DYNAMICAL MODEL}

First of all, a simple structural model of a BNNT can be considered in quite the same way as it was done for singlewalled carbon nanotubes (CNTs). ${ }^{20}$ In this model, a rectangular strip is cut out from an infinite $\mathrm{BN}$ honeycomb sheet perpendicular the line connecting two given lattice points. The strip is then rolled up into a seamless cylinder so that the two points coincide. Such a tube can uniquely be specified by the pair of integers $(n, m)$ that define the lattice translation vector between the two points. Such an ideal tube can also be characterized by its radius $R$ and chiral angle $\theta$, which is the angle between the tube circumference and the nearest zigzag of B-N bonds. The tubes are called achiral for $\theta=0$ (zigzag type) and $\theta=\pi / 6$ (armchair type), and chiral for $0<\theta<\pi / 6$. The ideal tube is a periodic structure along its axis with a primitive translation vector $T$ and has $N_{c}$ boron-nitrogen atomic pairs in the translational unit cell. The quantities $R, \theta, T$, and $N_{c}$ are given by the relations: $R$ $=\sqrt{3\left(n^{2}+n m+m^{2}\right)} a / 2 \pi, \quad \theta=\tan ^{-1}[\sqrt{3} m /(m+2 n)], \quad T$ $=2 \sqrt{3} \pi R / d$, and $N_{c}=2\left(n^{2}+n m+m^{2}\right) / d$ where $a$ is the nearest-neighbor B-N distance and $d$ is the greatest common divisor of $2 n+m$ and $2 m+n$.

Along with its translational periodicity, the ideal nanotube has a specific screw symmetry. As a consequence of the latter, a boron-nitrogen (B-N) atomic pair can be mapped onto the entire tube by use of two different screw operators, ${ }^{21}$ similar to the way in which the BN sheet is generated from such a pair by use of two primitive translation vectors. The lattice-dynamical model for a BNNT based on a two-atoms unit cell can then be constructed in the same way as for a 
three-dimensional periodic structure. ${ }^{22,23}$ For small displacements of the atoms from their equilibrium positions, the Hamiltonian of the nanotube can be written as the sum of the kinetic energy and the potential energy (in the harmonic approximations). Using this Hamiltonian, the equations of motion are readily derived. After Fourier transforming the atomic displacements and the force-constant matrix, the dynamical problem is reduced to the solution of a system of six homogeneous linear equations. The requirement for nontrivial solutions of these equations leads to the secular equation and, eventually, to the eigenvalues $\omega(q l j)$. Finally, the eigenvectors $\mathbf{e}(k \mid q l j)(k=1,2)$ are obtained as solutions of the equations of motion. Here the two quantum numbers $q$ and $l$ label the modes with a given wave number $q$ in the one-dimensional Brillouin zone of the tube $(-\pi \leqslant q \leqslant \pi)$ and with $2 l$ nodes of the eigenvector around the tube circumference $\left(l=0,1, \ldots, N_{c} / 2\right)$. The index $j(j=1,2, \ldots, 6)$ enumerates the modes with given $q$ and $l$. There is an exact correspondence between the modes with given $q$ and $l$ and the modes with given $k$ and $\mu$ obtained within the zonefolding method (see Eq. 14 in Ref. 19) only in the limit of very large tube radii. In that case, the phonon dispersion of a tube can be "unfolded" into the phonon dispersion of the BN sheet along the direction of the tube circumference by plotting it as a function of $l\left(l=0,1, \ldots, N_{c} / 2\right.$ and $\left.q=0\right)$.

The values of the quantum number $l$ correspond to the irreducible representations describing the symmetry properties of the phonons with respect to the symmetry groups of the tubes. ${ }^{24}$ Thus the $q=0$ phonons with $l=0,1, \ldots, N_{c} / 2$ transform according to the irreducible representations $A_{x}, E_{1 x}, E_{2 x}, \ldots, B_{x}(x=g$ or $u), A_{x}, E_{1}, E_{2}, \ldots, B_{x}(x$ $=1$ or 2 ), and $A, E_{1}, E_{2}, \ldots, B$ of the rod groups $C_{2 n h}$, $C_{2 n v}$, and $C_{N}$, in armchair, zigzag, and chiral tubes, respectively. The group-theoretical analysis based on these rod groups reveals that the Raman- and infrared-active phonons transform according to

$$
\begin{array}{cc}
\Gamma_{R}^{a r m}=3 A_{g} \oplus 2 E_{1 g} \oplus 4 E_{2 g}, & \Gamma_{i r}^{a r m}=A_{u} \oplus 3 E_{1 u}, \\
\Gamma_{R}^{z i g}=3 A_{1} \oplus 5 E_{1} \oplus 6 E_{2}, & \Gamma_{i r}^{z i g}=3 A_{1} \oplus 5 E_{1}, \\
\Gamma_{R}^{c h}=4 A \oplus 5 E_{1} \oplus 6 E_{2}, & \Gamma_{i r}^{c h}=4 A \oplus 5 E_{1} .
\end{array}
$$

Note that there are only eight, eight, and 14 Raman-active phonons and three, three, and six infrared-active phonons in armchair, zigzag, and chiral CNTs, respectively. ${ }^{25}$

It is important to point out that the necessary computational time for solving the eigenvalue problem for the twoatom unit cell is linear in $N_{c}$ while that for the $2 N_{c}$-atom translational unit cell increases as $N_{c}^{3}$. Thus this problem will be practically unsolvable for certain experimentally observable chiral nanotubes with large $N_{c}$ (note that among the $404 \mathrm{BN}$ tubes with radii between 5 and $15 \AA, 142$ tubes have $N_{c}>1000$ and 61 tubes have $N_{c}>2000$ ).
Here a valence shell model (VSM) based on a two-atom unit cell is used for the calculation of the phonon dispersion of BNNTs. In this model, the long-range interactions are described by Coulomb potentials and the short-range interactions are described by valence force field parameters of the types nearest-neighbor stretch $\alpha_{1}$, next-to-nearest-neighbor stretch $\alpha_{2}$, in-plane bend $\gamma_{1}$, and out-of-plane bend $\gamma_{2} \cdot{ }^{26}$ The out-of-plane bend interaction is necessary for the model because it stabilizes the out-of-plane dynamics that cannot be done alone by the Coulomb potentials. The electron density deformations of the boron and nitrogen ions are also accounted for representing each ion by a massless spherical shell with charge $Y$ coupled with a spring with constant $k$ to a point core with mass $m$ and charge $X(Z=X+Y$ is the ionic charge). The ionic polarizability is then $\alpha=Y^{2} / k$. The strong anisotropy of the $\mathrm{BN}$ sheets suggests to use two different values of the polarizability $\alpha_{\|}$and $\alpha_{\perp}$ for directions which are locally parallel and perpendicular to the tube surface, respectively. The existing experimental data on the tube structure is rather scarce. Ab initio structural studies of BNNTs have predicted buckling of the B-N bonds with a formation of a concentric inner $\mathrm{B}$ cylinder and an outer $\mathrm{N}$ cylinder. ${ }^{2}$ Since the separation between the two cylinders is $\sim 0.1$ a.u., the effect of the bond buckling on the phonons will be negligible. Therefore, a simple structural model was considered here, in which it was assumed that for a given tube all bonds are straight and of equal lengths of $1.44 \AA$ (as it is in the sheets of bulk h-BN), all bond angles are equal to each other, and all atoms lie on a single cylindrical surface.

Due to the large electronic band gap of the BNNTs of $\sim 5.5 \mathrm{eV}$, the Raman scattering of light is expected to be nonresonant. Therefore, a bond-polarizability model that is suitable to describe the nonresonant Raman intensity was used to calculate the Raman spectra of BNNTs. This model was applied here for three different tube types for backscattering geometry and parallel light polarization with averaging over all tube orientations in space. ${ }^{27}$

\section{RESULTS AND DISCUSSION}

The model parameters of any phenomenological lattice dynamical model are usually determined via fitting to available experimental data on the same or similar structures. In the case of BNNTs it is appropriate to derive the model parameters from data for graphite-like $\mathrm{BN}$ crystals. There are two such polymorphic structures. The most common one, h-BN, has $A A^{\prime}$ layer stacking with all hexagons in all layers coinciding but with boron and nitrogen atoms alternating from layer to layer at the same position. The unit cell of $\mathrm{h}-\mathrm{BN}$ contains two boron and two nitrogen atoms. The less common one, r-BN, has an $A B C$ layer stacking with the hexagons no longer coinciding but translated parallel to each other from one layer to the other. This structure is stable only at pressures $>10 \mathrm{GPa}$. A structure with layer stacking $\mathrm{AB}$ as in graphite does not exist for bulk BN. ${ }^{28}$ For this reason, the VSM parameters were derived here by fitting to available experimental data on h-BN, namely, the surface phonon dispersion curves of epitaxial monolayer films of h-BN measured by high-resolution energy-loss spectroscopy, ${ }^{29}$ Raman 
TABLE I. VSM parameters fitted to the measured phonon dispersion in Ref. 29.

\begin{tabular}{lcc}
\hline \hline Parameter ${ }^{\mathrm{a}}$ and unit & Symbol & Value \\
\hline Ionic charge (e) & $Z_{B}$ & 1.29 \\
& $Z_{N}$ & -1.29 \\
Shell charge (e) & $Y_{B}$ & 1.90 \\
& $Y_{N}$ & -1.90 \\
Ionic polarizability ${ }^{\mathrm{b}}\left(\AA^{3}\right)$ & $\alpha_{\|}$ & 0.01 \\
(for $B$ and $N$ ) & $\alpha_{\perp}$ & 0.61 \\
& $\alpha_{1}(B-N)$ & 610.8 \\
Nearest-neighbor stretch $(\mathrm{N} / \mathrm{m})$ & $\alpha_{2}(B-B)$ & 23.6 \\
Next-to-nearest neighbor & $\alpha_{2}(N-N)$ & 2.5 \\
stretch $(\mathrm{N} / \mathrm{m})$ & $\gamma_{1}(B-N-B)$ & 29.5 \\
& $\gamma_{1}(N-B-N)$ & -10.0 \\
In-plane bend $\left(10^{-20} \mathrm{Nm}\right)$ & $\gamma_{2}$ & 14.8 \\
Out-of-plane bend $\left(10^{-20} \mathrm{Nm}\right)$ & & \\
(for $B$ and $N$ ) & & \\
\hline
\end{tabular}

${ }^{\mathrm{a}}$ The repulsion between $\mathrm{B}$ and $\mathrm{N}$ atoms of neighboring sheets is described by a Born-Mayer potential $V(r)=a \exp (-b r)$ with $a$ $=638 \mathrm{eV}$ and $b=3.18 \AA^{-1}$.

${ }^{\mathrm{b}}$ Anisotropic ionic polarizability was assumed for both $\mathrm{B}$ and $\mathrm{N}$. The symbols $\|$ and $\perp$ denote directions which are parallel and perpendicular to the sheets, respectively.

scattering and infrared absorption data on bulk h-BN. ${ }^{30}$ The best-fit VSM parameters are given in Table I. The in-plane polarizabilities $\alpha_{\|}$were found to be vanishingly small, while finite values were necessary for the out-of-plane ones $\alpha_{\perp}$ in order to reach agreement with the experimental data. The fitting procedure yielded for each of the quantities $\alpha_{\|}$and $\alpha_{\perp}$ nearly the same values for B and N. The out-of-plane bending parameters for $\mathrm{B}$ and $\mathrm{N}$ were assumed equal because different values of these parameters do not improve much the disagreement with experiment for the out-of-plane phonon branches.

The calculated phonon dispersion curves are shown in comparison to the experimental points in Fig. 1. The in-plane acoustic and optical phonon curves, denoted by LA, TA $A_{\|}$, $\mathrm{LO}$, and $\mathrm{TO}_{\|}$, could be reproduced quite well while there is a clear disagreement for the out-of-plane phonon curves $\mathrm{TA}_{\perp}$ and $\mathrm{TO}_{\perp}$. The reason for this can be found in the high anisotropy of bulk h-BN along and perpendicular to the $\mathrm{BN}$ sheets. Due to the layered structure of h-BN, the separate layers must have out-of-plane phonons with a nearly quadratic wave-vector dependence of their frequency. However, such dependence cannot be reproduced by use of two-body interaction force constants but requires the introduction of many-body ones. In the case of a graphite sheet, the quadratic dependence could only be described by the introduction of out-of-plane bending and bond-twisting valence force field parameters. ${ }^{31}$ This set of parameters allowed one to predict a quadratic wave-vector dependence for the transverse acoustic branches of carbon nanotubes. ${ }^{22}$ In the case of a BN sheet, one has to account for the long-range interactions by use of Coulomb potentials which rules out the possibility of

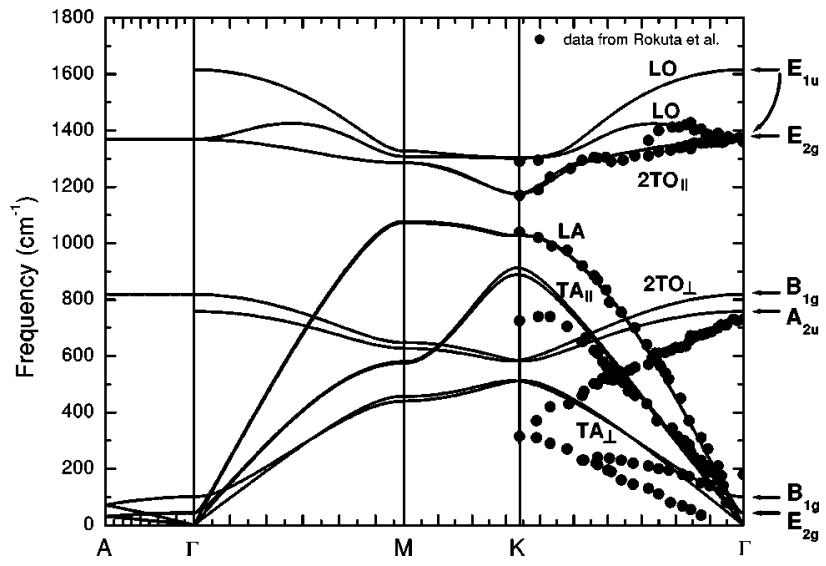

FIG. 1. Phonon dispersion curves of bulk h-BN along several high-symmetry directions calculated within a valence shell model with parameters fitted to the experimental data from Refs. 29 and 30 . The type of the phonon branches along $\Gamma K$ direction and the symmetry of the optical phonons are given as well.

a derivation of a quadratic dependence, and the resulting dependence will only be linear in the wave vector. It is worth mentioning that such problems do not arise in ab initio dynamical calculations of h-BN, possibly due to the many-body character of the long-range interactions there. ${ }^{32}$ The mentioned drawback of our model is not expected to influence the results for the high-intensity Raman-active phonons of a BN tube much because they are in-plane stretching phonons ( $G$-band phonons) or are determined by bond-stretching interactions [the radial breathing mode (RBM)]. For these reasons, only the experimental points for the in-plane phonons together with the two optical out-of-plane phonon frequencies were accounted for in the fitting procedure. In Table II, the calculated frequencies and vibrational patterns of the zone-center optical phonons of bulk h-BN are given together with the observed ones. ${ }^{30}$

The derived model parameters can be used to calculate the phonon dispersion of various BNNTs. The phonon dispersion of tubes $(10,10),(17,0)$, and $(15,4)$, with $N_{c}=20$, 34 , and 602 , respectively, and nearly the same radius of $\approx 6.8 \AA$, are presented in Fig. 2 . The branches with $l$ $=0,1,2$ are drawn by solid lines and those with $l$ $=3,4, \ldots, N_{c} / 2$ are drawn by dotted lines or are omitted as in the case of tube $(15,4)$. The symmetry of the Raman- and infrared-active phonons is shown as well. The zone-center phonons of the tubes $(10,10)$ and $(17,0)$ are given in a unfolded graph in Fig. 3. For armchair and zigzag tubes with very large radii, the unfolded curves will coincide with the corresponding curves for the $\mathrm{BN}$ sheet along the directions $\Gamma M$ and $\Gamma K$, respectively. In the case of armchair and zigzag tubes with finite radii there will still be a similarity between the unfolded curves and the sheet dispersion along $\Gamma M$ and $\Gamma K$. Thus the $\Gamma$-point phonons of the sheet are displacements identical for all boron-nitrogen pairs, while those for $\mathbf{q} \neq \mathbf{0}$ are standing waves along $\mathbf{q}$. Similarly, the zone-center tube phonons with $l=0$ are displacements identical for all BN pairs with respect to the tube surface and those with $l$ $\neq 0$ are standing waves with $2 l$ nodes around the 
TABLE II. Calculated and measured fequencies of the zone-center optical phonons of bulk h-BN.

\begin{tabular}{lccc}
\hline \hline Optical phonon $^{\mathrm{a}}$ & Symmetry & Obs. ${ }^{30}$ & Calc. \\
\hline antisymmetric counterphase in-plane & $E_{1 u}($ TO/LO $)$ & $1367 / 1610$ & $1367 / 1612$ \\
symmetric counterphase in-plane & $E_{2 g}$ & 1366 & 1366 \\
antisymmetric counterphase out-of-plane & $A_{2 u}(T O / L O)$ & $783 / 828$ & $758 / 819$ \\
symmetric counterphase out-of-plane & $B_{1 g}$ & - & 819 \\
symmetric in-phase out-of-plane & $B_{1 g}$ & - & 100 \\
symmetric in-phase in-plane & $E_{2 g}$ & 52 & 43 \\
\hline \hline
\end{tabular}

"The descriptions "in-phase" and "counter-phase" refer to the dispacements of B and N on the same BN sheet, and "symmetric" and "antisymmetric" characterize the atomic displacements with respect to the center of symmetry of h-BN.

circumference. $^{22}$ For $q=0$ and $l=0$, there are two acoustic phonons with equal displacements of all atoms along the tube axis and along the circumference (the two zerofrequency transverse acoustic phonons have $l=1$ ) and four optical phonons: one with uniform in-phase radial displacements of all atoms (the RBM), another with counterphase radial displacements of all atoms, and two with counterphase in-plane phonons. The phonons of the "acoustic" and "optical" branches in Fig. 3 are in-phase and counterphase motions of the unit cell pair in the form of sinosuidal standing waves with $2 l$ nodes around the tube circumference.

The zone-center Raman- and infrared-active phonons are important for the structural characterization of the BNNT samples. In the case of CNTs, the RBM and the in-plane stretching modes forming the so-called $G$ band have the highest Raman intensity. These modes are expected to give rise to high-intensity Raman peaks for BNNTs as well. Here the dependence of the RBM frequency (in $\mathrm{cm}^{-1}$ ) on the radius $R$ (in $\AA$ ) (see Fig. 4) was fitted with a simple power law:
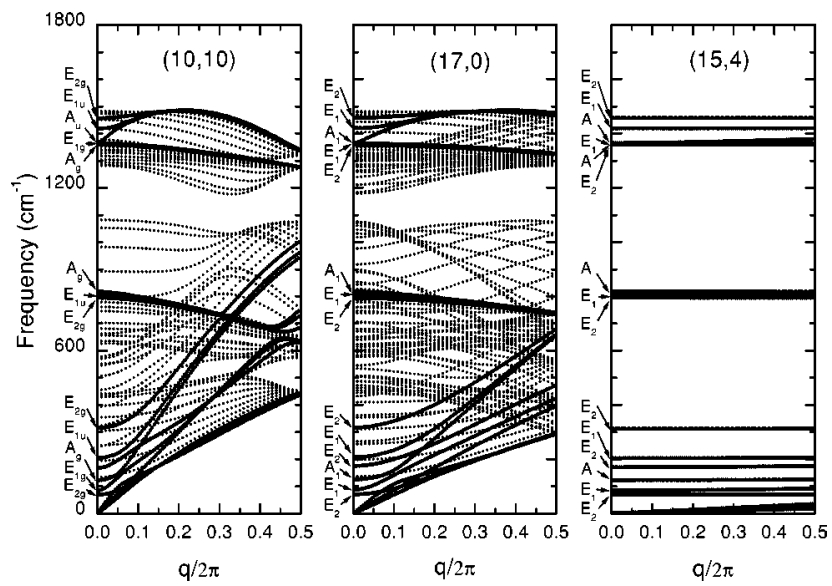

FIG. 2. Calculated phonon dispersion curves for the tubes $(10,10),(17,0)$, and $(15,4)$ of armchair, zigzag, and chiral types, respectively. The branches with $l=0,1,2$ are drawn with continuous lines and those for $l=3,4, \ldots, N_{c} / 2$ are drawn with dotted lines or are omitted. The symmetry of the Raman- and infrared-active phonons is given as well.

$$
\omega(\mathrm{RBM})=\frac{851}{R} .
$$

In the case of CNTs, the force-constant approach yields values for the RBM frequency which are lower by a few percent than the experimental ones [cf., e.g., 1148/R (Ref. 22) to $1240 / R$ (Ref. 33)]. Therefore, it is expected that this formula will underestimate the actual RBM frequency. The radius dependence of the frequencies of the $G$-band modes for all 1189 tubes with radii from 5 to $25 \AA$ is shown in Fig. 5. With the increase of the radius, all curves tend asymptotically to the frequency of the $\mathrm{BN}$ sheet stretching mode of $1367 \mathrm{~cm}^{-1}$. The curves were fitted with simple power laws (with respect to the frequency $1367 \mathrm{~cm}^{-1}$ ): $-115 / R^{2.11}$ and $-199 / R^{1.96}$ ( $A$ modes), $272 / R^{0.85}$ and $-155 / R^{1.94}\left(E_{1}\right.$ modes), and $382 / R^{0.75}$, and $-364 / R^{1.95}$ ( $E_{2}$ modes). The softening/hardening of the frequencies of the $G$-band modes is entirely a curvature effect in the current dynamical simulations. Electronic band structure effects, which are signifi-

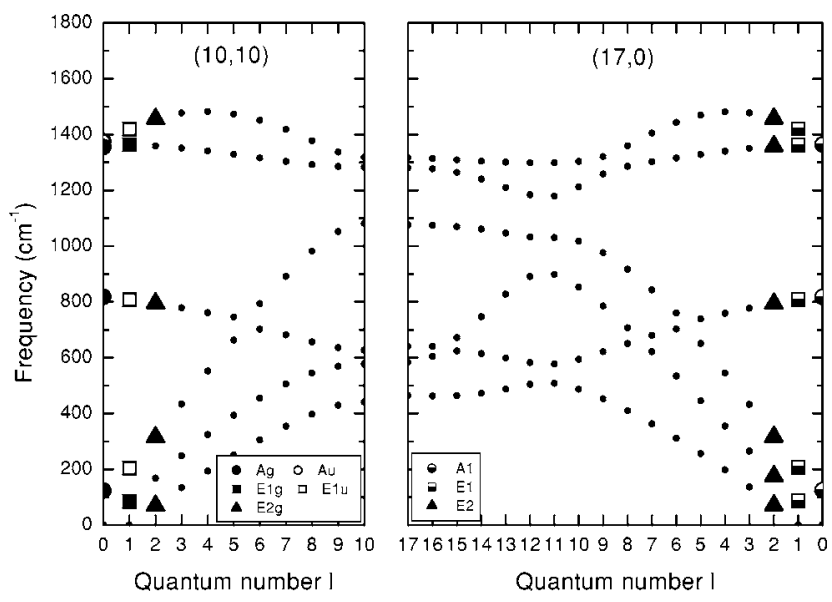

FIG. 3. Unfolded phonon dispersion for the zone-center phonons of the tubes $(10,10)$ and $(17,0)$. The Raman-active (infrared-active) modes with $l=0,1,2$ are given by solid (empty) circles, squares and triangles. The semisolid symbols denote modes which are both Raman and infrared active. The symmetry of these phonons is provided in the legends. 


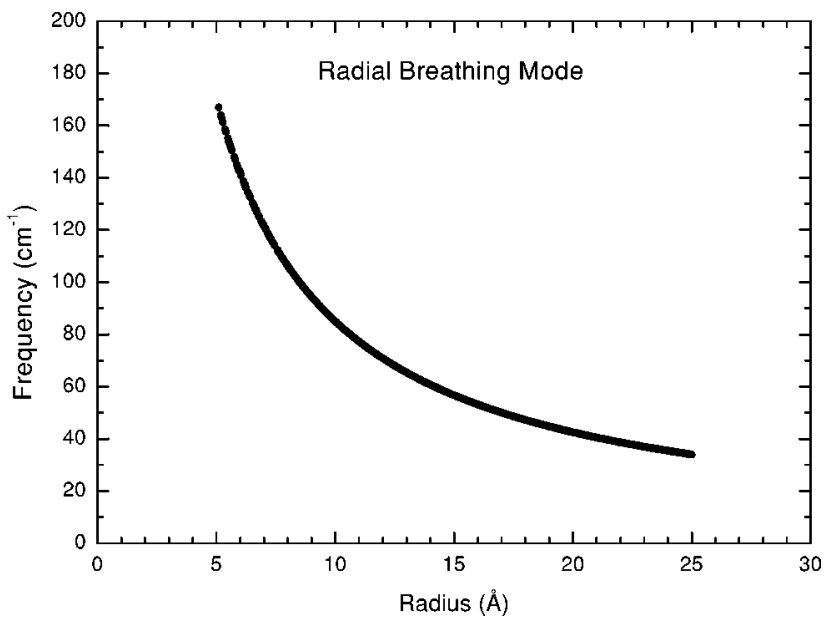

FIG. 4. Calculated radius dependence of the frequency of the radial breathing mode for all 1189 tubes (chiral and achiral) with radii between 5 and $25 \AA$. The curve was fitted with a simple power law (see the text for details).

cant in CNTs, ${ }^{34}$ can be ruled out for BNNTs due to the large electronic band gaps.

The assignment of the peaks in the observed Raman spectra can essentially be facilitated if the Raman intensity can be predicted as well. Due to the large electronic band gaps of BNNTs, the resonant Raman scattering effects are relatively weak. For this reason, the calculation of the Raman intensity is carried out here within a nonresonant model, namely, a bond-polarizability model. Since there are no available polarizabilities and their derivatives for the B-N bonds, the calculation was done with parameters for the $\mathrm{C}-\mathrm{C}$ bonds. ${ }^{27}$ The Raman intensity of the $G$-band modes was found to be in-

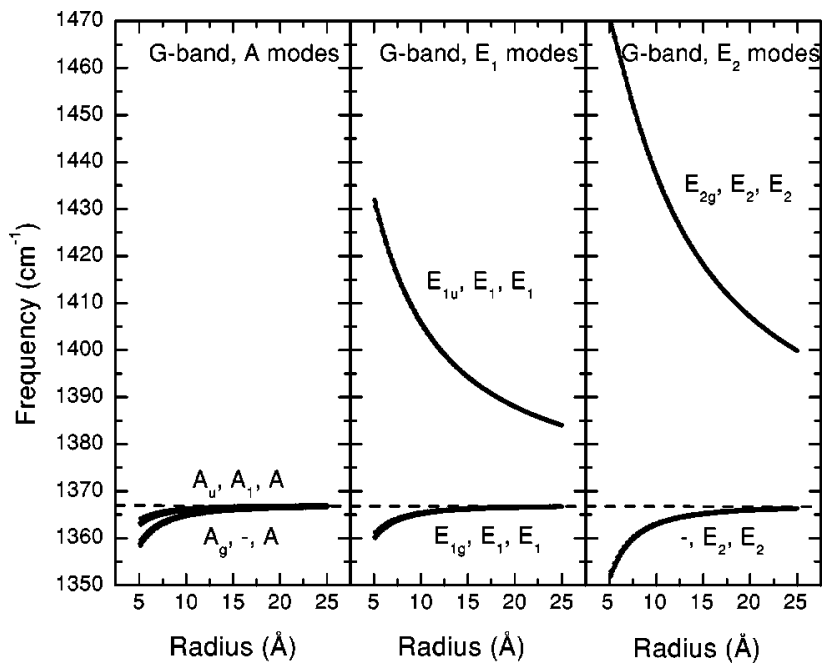

FIG. 5. Calculated radius dependence of the frequency of the six in-plane stretching optical modes with $l=0,1,2$ for all 1189 tubes with radii between 5 and $25 \AA$. The three symmetry species at each of the curves refer to tubes of armchair, zigzag, and chiral types, respectively. The dashes mean that for the corresponding tube types the modes are neither Raman active nor infrared active [see Eqs. (2.1)-(2.3)].

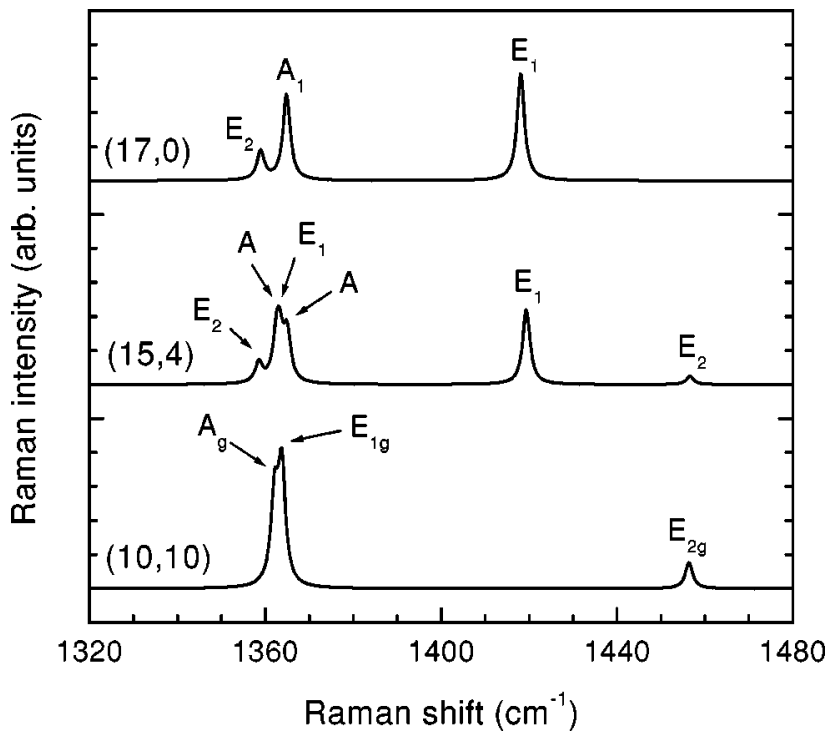

FIG. 6. Calculated Raman spectra for the three tubes $(10,10)$, $(17,0)$, and $(15,4)$. Full width at half maximum of $1 \mathrm{~cm}^{-1}$ was assumed for all lines. The symmetry and position of the Ramanactive lines is shown as well.

sensitive to large variations of the bond polarizability parameters, while the intensity of the RBM changed by several orders of magnitude for the same parameters variations. This justifies the presentation of the $G$-band region of the Raman spectra mainly for illustration that certain Raman-active modes give rise to significant intensity and other Ramanactive modes have vanishingly small intensity. The highfrequency regions of the calculated spectra containing the lines of the $G$-band modes for the three tubes $(10,10)$, $(17,0)$, and $(15,4)$ are shown in Fig. 6 . It is seen that the modes with $A$ and $E_{1}$ symmetry give rise to higher peaks that the $E_{2}$ modes. Several peaks are closely situated at about $1370 \mathrm{~cm}^{-1}$ and they could be seen as a single peak. However, the observation of the $E_{1}$ peak at $1419 \mathrm{~cm}^{-1}$ or the $E_{2}$ peak at $1457 \mathrm{~cm}^{-1}$ will be a signature for the presence of tubes $(10,10)$ or $(17,0)$, respectively. In the case of a BNNT sample with a certain distribution of the tube radii, the assignment of the Raman lines can be done by use of Fig. 5 and the calculated Raman intensity. The latter data are not given here because the Raman intensity of the $G$ modes as a function of the tube radius and chirality cannot be fitted with smooth curves. In the case of the RBM, however, the product of the intensity and the volume per boron-nitrogen pair is approximately a constant which is independent of the tube radius and chirality. The RBM intensity itself will depend on the choice of the unit cell volume (for a discussion, see Ref. 22).

\section{CONCLUSIONS}

In conclusion, the lattice dynamics of single-walled boron nitride nanotubes is calculated within a valence shell model. The results for the phonon dispersion for three different tubes are presented. Special attention is given to the study of 
the zone-center phonons. The nonresonant Raman intensity for an armchair, a zigzag and a chiral tube is calculated within a bond-polarizability model. The obtained frequencies of the radial breathing mode and the in-plane stretching optical phonons of the $G$ band as a function of the tube radius can be used for the assignment of the Raman and infrared spectra of BNNT samples.

\section{ACKNOWLEDGMENTS}

The author acknowledges the financial support by the Flemish Region-IWT and by the NATO Research Program for Belgium. He also thanks the University of Antwerp (RUCA) for its support in the framework of the Visiting Professors Program.
*Permanent address: Faculty of Physics, University of Sofia, BG1164 Sofia, Bulgaria.

${ }^{1}$ A. Rubio, J. L. Corkill, and M. L. Cohen, Phys. Rev. B 49, 5081 (1994).

${ }^{2}$ X. Blase, A. Rubio, S. G. Louie, and M. L. Cohen, Europhys. Lett. 28, 335 (1994).

${ }^{3}$ N. G. Chopra, R. J. Luyken, K. Cherrey, V. H. Crespi, M. L. Cohen, S. G. Louie, and A. Zettl, Science 69, 966 (1995).

${ }^{4}$ A. Loiseau, F. Willaime, N. Demoncy, G. Hug, and H. Pascard, Phys. Rev. Lett. 76, 4737 (1996).

${ }^{5}$ M. Terrones, W. K. Hsu, H. Terrones, J. P. Zhang, S. Ramos, J. P. Hare, R. Castillo, K. Prassides, A. K. Cheetham, H. W. Kroto, and D. R. M. Walton, Chem. Phys. Lett. 259, 568 (1996).

${ }^{6}$ Y. Saito, M. Maida, and T. Mastumoto, Jpn. J. Appl. Phys. 38, 159 (1999).

${ }^{7}$ D. Golberg, Y. Bando, M. Eremets, K. Takemura, K. Kurashima, and H. Yusa, Appl. Phys. Lett. 69, 2045 (1996).

${ }^{8}$ D. P. Yu, X. S. Sun, C. S. Lee, I. Bello, S. T. Lee, H. D. Gu, K. M. Leung, G. W. Zhou, Z. F. Dong, and Z. Zhang, Appl. Phys. Lett. 72, 1966 (1998).

${ }^{9}$ O. R. Lourie, C. R. Jones, B. M. Bartlett, P. C. Gibbons, R. S. Ruoff, and W. E. Buhro, Chem. Mater. 12, 1808 (2000).

${ }^{10}$ M. Terauchi, M. Tanaka, K. Suzuki, A. Ogino, and K. Kimura, Chem. Phys. Lett. 324, 359 (2000).

${ }^{11}$ B. G. Demczyk, J. Cumings, A. Zettl, and R. O. Ritchie, Appl. Phys. Lett. 78, 2772 (2001).

${ }^{12}$ R. Ma, Y. Bando, T. Sato, and K. Kurashima, Chem. Mater. 13, 2965 (2001).

${ }^{13}$ C. C. Tang, M. Lamy de la Chapelle, P. Li, Y. M. Liu, H. Y. Dang, and S. S. Fan, Chem. Phys. Lett. 342, 492 (2001).

${ }^{14}$ R. S. Lee, J. Gavillet, M. Lamy de la Chapelle, A. Loiseau, J.-L. Cochon, D. Pigache, J. Thibault, and F. Willaime, Phys. Rev. B 64, 121405 (2001).

${ }^{15}$ N. G. Chopra and A. Zettl, Solid State Commun. 105, 297 (1998).

${ }^{16}$ X. Blase, A. DeVita, J.-C. Charlier, and R. Car, Phys. Rev. Lett. 80, 1666 (1998).
${ }^{17}$ E. Hernandez, C. Goze, P. Bernier, and A. Rubio, Phys. Rev. Lett. 80, 4502 (1998).

${ }^{18}$ M.-F. Yu, O. Lourie, M. J. Dyer, K. Moloni, T. F. Kelly, and R. S. Ruoff, Science 287, 637 (2000).

${ }^{19}$ M. S. Dresselhaus and P. C. Eklund, Adv. Phys. 49, 705 (2000).

${ }^{20}$ R. Saito, G. Dresslhaus, and M. S. Dresselhaus, Physical Properties of Carbon Nanotubes (Imperial College Press, London, 1998).

${ }^{21}$ C. T. White, D. H. Robertson, and J. W. Mintmire, Phys. Rev. B 47, 5485 (1993).

${ }^{22}$ V. N. Popov, V. E. Van Doren, and M. Balkanski, Phys. Rev. B 59, 8355 (1999); 61, 3078 (2000).

${ }^{23}$ J. Maultzsch, S. Reich, C. Thomsen, E. Dobardžić, and M. Damnjanović, Solid State Commun. 121, 471 (2002).

${ }^{24}$ O. E. Alon, Phys. Rev. B 64, 153408 (2001).

${ }^{25}$ O. E. Alon, Phys. Rev. B 63, 201403 (2001).

${ }^{26}$ E. B. Wilson, Jr., J. C. Decius, and P. C. Cross, Molecular Vibrations (Dover, New York, 1955), Chap. 4.

${ }^{27}$ R. Saito, T. Takeya, T. Kimura, G. Dresselhaus, and M. S. Dresselhaus, Phys. Rev. B 57, 4145 (1998).

${ }^{28}$ J. H. Edgar, in Properties of Group III Nitrides, edited by J. H. Edgar, EMIS Datareviews Series No 11 (INSPEC, London, 1994), p. 7.

${ }^{29}$ E. Rokuta, Y. Hasegawa, K. Suzuki, Y. Gamou, C. Oshima, and A. Nagashima, Phys. Rev. Lett. 79, 4609 (1997).

${ }^{30}$ R. Geick, C. H. Perry, and G. Rupprecht, Phys. Rev. 146, 543 (1966).

${ }^{31}$ T. Aizawa, R. Souda, S. Otani, Y. Ishizawa, and C. Oshima, Phys. Rev. B 42, 11469 (1990); 43, 12060(E) (1991).

${ }^{32}$ G. Kern, G. Kresse, and J. Hafner, Phys. Rev. B 59, 8551 (1999).

${ }^{33}$ A. Jorio, R. Saito, J. H. Hafner, C. M. Lieber, M. Hunter, T. McClure, G. Dresselhaus, and M. S. Dresselhaus, Phys. Rev. Lett. 86, 1118 (2001).

${ }^{34}$ M. S. Dresselhaus, G. Dresselhaus, A. Jorio, A. G. Souza Filho, and R. Saito, Carbon 40, 2043 (2002). 